\title{
Intense mass loss from C-rich AGB stars at low metallicity?
}

\author{
L. Mattsson, R. Wahlin, S. Höfner, and K. Eriksson
}

\author{
Dept. Physics and Astronomy, Div. of Astronomy and Space Physics, Uppsala University, Box 515, 75120 Uppsala, Sweden \\ e-mail: mattsson@astro.uu.se
}

Received 29 February 2008 / Accepted 15 April 2008

ABSTRACT

\begin{abstract}
We argue that the energy injection of pulsations may be of greater importance to the mass-loss rate of AGB stars than metallicity, and that the mass-loss trend with metallicity is not as simple as sometimes assumed. Using our detailed radiation hydrodynamical models that include dust formation, we illustrate the effects of pulsation energy on wind properties. We find that the mass-loss rate scales with the kinetic energy input by pulsations as long as a dust-saturated wind does not occur, and all other stellar parameters are kept constant. This includes the absolute abundance of condensible carbon (not bound in CO), which is more relevant than keeping the C/O-ratio constant when comparing stars of different metallicity. The pressure and temperature gradients in the atmospheres of stars, become steeper and flatter, respectively, when the metallicity is reduced, while the radius where the atmosphere becomes opaque is typically associated with a higher gas pressure. This effect can be compensated for by adjusting the velocity amplitude of the variable inner boundary (piston), which is used to simulate the effects of pulsation, to obtain models with comparable kinetic-energy input. Hence, it is more relevant to compare models with similar energy-injections than of similar velocity amplitude. Since there is no evidence for weaker pulsations in low-metallicity AGB stars, we conclude that it is unlikely that low-metallicity C-stars have lower mass-loss rates, than their more metal-rich counterparts with similar stellar parameters, as long as they have a comparable amount of condensible carbon.
\end{abstract}

Key words. stars: AGB and post-AGB - stars: atmospheres - stars: carbon - stars: mass-loss - hydrodynamics - radiative transfer

\section{Introduction}

According to the most commonly accepted scenario, the mass loss of AGB stars is driven by radiation pressure on dust-grains in the outer stellar atmospheres. This pressure causes the acceleration of the outer layers, which develop, as the escape-velocity is reached, into a stellar wind. This mechanism is enhanced by the pulsations of these stars and the resulting shock waves which create conditions for efficient dust condensation. The wind momentum is, however, generated by radiative acceleration, since pulsations alone cannot inject sufficient energy to sustain an outflow (Wood 1979).

The metallicity of an AGB star can affect its wind properties since it influences the atmospheric structure and possibly the availability of condensible material for dust formation. Whether low metallicity should be associated with smaller mass loss is however unclear. Bowen \& Willson (1991) argued that lowmetallicity AGB stars should experience a so-called superwind phase, but proposed that this should occur at a higher luminosity than for a metal-rich case. Their argument was that at lower metallicities the molecular opacities are smaller, which in turn can reduce the extension of the atmosphere. Since the onset of dust formation depends on whether the atmosphere has reached a critical size, it appears correct to expect that weaker winds occur at lower metallicities. On the other hand, observational studies of metal-poor carbon stars in the Magellanic Clouds and the Milky Way indicate similar mass-loss rates (van Loon et al. 2000; Groenewegen et al. 2007).

We report results of an on going project to explore the mass loss properties of C-rich AGB stars as a function of stellar parameters. We question the common idea that AGB stars at lower metallicity should have weaker winds, and argue that the injection of kinetic energy by pulsations plays an important rôle in wind formation and the resultant mass-loss rate. We claim that commonly-made assumptions about boundary conditions in mass-loss models of AGB stars make it difficult to compare results in the literature. The simulation of stellar pulsations, using a time-dependent inner boundary condition, requires, in particular, a careful choice of parameterisation; for example, results derived using different rates of kinetic-energy injection, should not be compared.

Theoretical studies of dust-driven AGB mass loss at subsolar (LMC) metallicity (Wachter et al. 2008), suggested that these stars should have slower winds, but that the mass-loss rate should be mostly unaffected by the decrease in metallicity. In these models, a constant $\mathrm{C} / \mathrm{O}=1.8$ was assumed, which was the same value as in the solar-metallicity models of Wachter et al. (2002). Due to this assumption, the LMC-models have a significantly lower abundance of free carbon, i.e., carbon that is not bound to $\mathrm{CO}$ and may therefore form dust particles. As a consequence, less dust forms. A similar effect was observed in the results by Helling et al. (2000), who found that models of LMC metallicity have, in general, slower winds and a lower mass-loss rate. Given the assumptions, these trends are unsurprising, since reducing the amount of free carbon, and consequently the dust/gas-ratio, makes momentum transfer from radiation to dust and gas less efficient, leading to a slower wind. We note that molecular bands in metal-poor C-stars are as strong as, or stronger than the corresponding stars at solar metallicity. This was first observed in the optical (Westerlund 1991) and recently confirmed in the infrared (see, e.g., Lagadec et al. 2007, and references therein. The wind models presented by Wachter et al. (2008) and Helling et al. (2000) are also based on grey ra diative 
transfer, which appears to affect the mass-loss rates cf. Höfner (2003). Moreover, the velocity amplitudes and not the rates of energy-injection by pulsations were used to quantify the effects of pulsations on mass loss (see, also, Winters et al. 2000).

\section{Numerical modelling}

The models for the atmospheres and winds are computed using frequency dependent radiative transfer for the gas and dust, including detailed micro-physics of the dust grains and their formation (described in Andersen et al. 2003; Höfner et al. 2003), in combination with time-dependent hydrodynamics. Here we describe the initial and boundary conditions, in addition, to further assumptions.

\subsection{Pulsations and the inner boundary condition}

The kappa-mechanism (see, e.g., Olivier \& Wood 2005), which is believed to be the cause of long-period pulsations, operates in the convective envelope. In our models, we simulate the effect of this mechanism on the atmosphere, using a time-dependent inner boundary condition, i.e., a so-called piston boundary of the form

$R_{\text {in }}(t)=R_{\text {in }}(0)+\frac{\mathcal{P}}{2 \pi} \Delta u_{\mathrm{p}} \sin \left(\frac{2 \pi}{\mathcal{P}} t\right)$,

where $\Delta u_{\mathrm{p}}$ is the velocity amplitude and $\mathcal{P}$ is the pulsation period. We employ an empirical period-luminosity relation (Feast et al. 1989), which maintains a direct relationship between the period and luminosity, for all models. Since the bottom layers of the atmosphere must be optically thick for the model atmosphere to be realistic, one should place the piston boundary in that optically thick region of the atmosphere. This leads to inner boundary conditions for the metal-poor (SMC-like) models where the gas pressure is significantly higher at $R=R_{\text {in }}$ (see Fig. 1), while the temperature changes much less. Thus, the kinetic energy-injection by the pulsations/piston is typically larger in these models for a given $\Delta u_{\mathrm{p}}$.

\subsection{Free carbon and dust formation}

The raw-material for dust grains is the condensible carbon in the atmosphere, i.e., the carbon that is not bound in $\mathrm{CO}$ molecules and therefore, in principle, available for dust formation. Since the absolute oxygen abundance $\varepsilon_{\mathrm{O}}$ may vary depending on the initial metallicity of the star and the amount of oxygen dredgeup, $\tilde{\varepsilon}_{\mathrm{C}} \approx \varepsilon_{\mathrm{C}}-\varepsilon_{\mathrm{O}}$ is a more relevant quantity than $\mathrm{C} / \mathrm{O}=\varepsilon_{\mathrm{C}} / \varepsilon_{\mathrm{O}}$.

Low-metallicity $\mathrm{C}$-stars do not necessarily have carbon-poor atmospheres. Since oxygen (along with large amounts of carbon) is most likely dredged-up in each thermal-pulse cycle (see, e.g., Herwig 2005, and references therein) the C/O-ratios do not have to be large, even if $\tilde{\varepsilon}_{\mathrm{C}}$ is relatively large. For C-stars with a solar (or higher) relative abundance of oxygen, the oxygen abundance is hardly affected by oxygen dredge-up, but for metal-poor C-stars, where the inital abundance is small, it certainly is. We emphasise that observations by Wahlin et al. (2006) indicate that $\mathrm{C} / \mathrm{O}$-ratios in metal-poor C-stars may be almost an order of magnitude higher than at solar metallicity; so even without oxygen dredge-up, $\tilde{\varepsilon}_{C}$ may therefore be quite large. After a number of thermal pulses, there may be as much free carbon in a metalpoor C-star atmosphere as for a solar-metallicity.

We assume that $\tilde{\varepsilon}$ is almost unrelated to the over all initial metallicity of the star. We use the same $\tilde{\varepsilon}$ for subsolar and solar-metallicity models, i.e., we compare the effects of different
Table 1. CNO abundances and metallicity of the models considered in this letter.

\begin{tabular}{lccccr}
\hline \hline & $\log \left(\tilde{\varepsilon}_{\mathrm{C}}\right)$ & $\log \left(\varepsilon_{\mathrm{C}}\right)$ & $\log \left(\varepsilon_{\mathrm{N}}\right)$ & $\log \left(\varepsilon_{\mathrm{O}}\right)$ & {$[\mathrm{Fe} / \mathrm{H}]$} \\
\hline AGS05 & $8.50 / 8.80$ & $8.89 / 9.04$ & 7.78 & 8.66 & 0.0 \\
Sub-solar & $8.50 / 8.80$ & $8.81 / 8.86$ & 6.78 & 7.96 & -1.0 \\
\hline
\end{tabular}

Table 2. Parameters used for the models. All models have $M_{\star}=1 M_{\odot}$, A-models have $\log (\tilde{\varepsilon})=8.80$, and B-models $\log (\tilde{\varepsilon})=8.50$.

\begin{tabular}{cccccc}
\hline \hline Model & $\log \left(L / L_{\odot}\right)$ & $T_{\text {eff }}(\mathrm{K})$ & $\log (g)$ & $\mathcal{P}(\mathrm{d})$ & $\Delta u_{\mathrm{p}}\left(\mathrm{km} \mathrm{s}^{-1}\right)$ \\
\hline A1 & 3.85 & 2800 & -0.65 & 390 & 3.0 \\
A2 & 3.85 & 2800 & -0.65 & 390 & 4.0 \\
A3 & 3.85 & 2800 & -0.65 & 390 & 5.0 \\
B1 & 3.70 & 2600 & -0.64 & 294 & 4.0 \\
B2 & 3.70 & 2600 & -0.64 & 294 & 5.0 \\
B3 & 3.70 & 2600 & -0.64 & 294 & 6.0 \\
\hline
\end{tabular}

metallicities only. Since $\tilde{\varepsilon}$, and not the C/O-ratio, remains fixed, it is not obvious that a lower initial metallicity leads to lower mass-loss rates, when comparing mass-loss models at different metallicities.

\section{Results and discussion}

\subsection{Numerical results}

We computed a set of models, where we considered two sets of stellar parameters (labelled A and B), two chemical compositions (see Tables 1 and 2), and various positions of the inner (piston) boundary. The results are the following (see also Figs. 2 and 3). For a fixed value of $\tilde{\varepsilon}_{\mathrm{C}}$ and a given set of stellar parameters, we found that:

- The location of the inner boundary can affect significantly the mass-loss rate if $\Delta u_{\mathrm{p}}$ is kept fixed.

- Models with a metal-poor composition (apart from carbon) have similar, or higher, mass-loss rates compared to models of solar composition (defined as that of Asplund et al. 2005) if the kinetic-energy injections by pulsations are similar.

- Models with a solar composition, in general, show slower winds, lower mass-loss rates, and lower degrees of dust condensation than models with metal-poor composition for fixed $\Delta u_{\mathrm{p}}$ if the inner boundary is located at smiliar optical depth, due to the difference in density at that point.

We emphasise that we compare models with the same absolute abundance of free carbon, which excludes the possibility that these effects are due to the accessibility of raw material for dust formation (see Sect. 2.2 for further details). However, the different absolute abundances of elements other than carbon affect molecular opacities and, consequently, the structure of the atmosphere. Compared to these abundance differences, the difference in the mass-loss properties of both of the models considered (A and B), appears to be more strongly correlated with the input of kinetic energy at the inner boundary. In our models, we use a sinusoidal piston boundary condition with a velocity amplitude $\Delta u_{\mathrm{p}}$, which means that the energy injected into a mass shell of mass $\mathrm{d} m$ at the inner boundary is $\mathrm{d} E_{\mathrm{p}}=\frac{1}{2} \Delta u_{\mathrm{p}}^{2} \mathrm{~d} m$. From an observational point of view, the mass loss rate appears to be proportional to the inferred pulsational energy injection rate of AGB stars (see van Loon 2008, and references therein). We therefore define a quantity

$q \equiv \Delta u_{\mathrm{p}}^{2} \rho_{\text {in }}(t=0)$ 

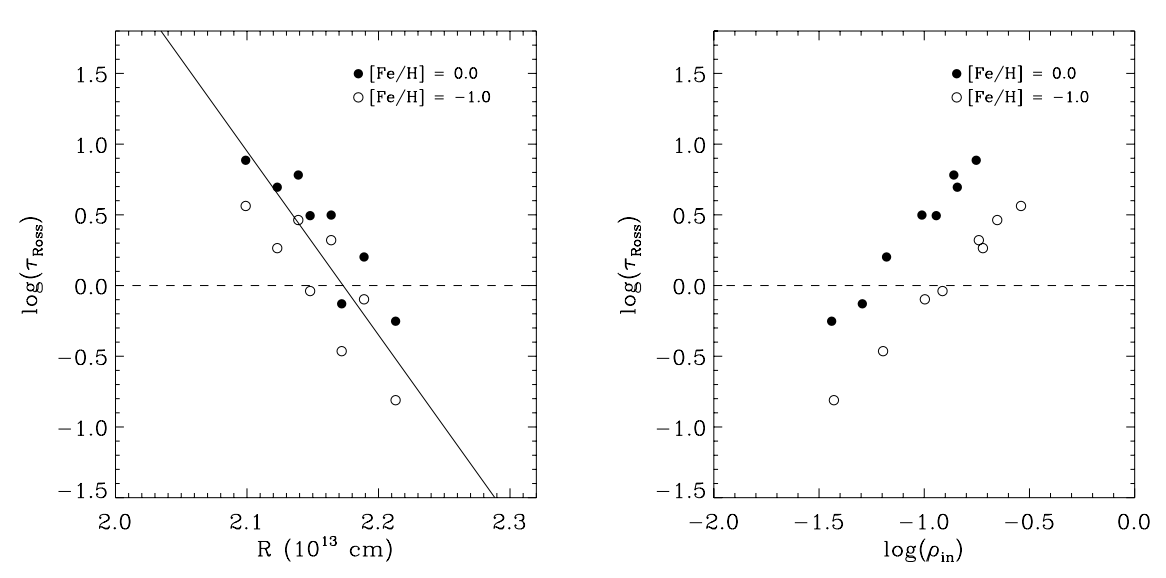

Fig. 1. The Rosseland mean opacity at the inner boundary of the hydrostatic initial atmospheric structures as function of the geometric location of the boundary and the gas density at the boundary (at $t=0$ ) for solar (Asplund et al. 2005) and a typical metal-poor composition $([\mathrm{Fe} / \mathrm{H}]=-1.0)$. The full line in the left panel shows an opacity-law of the form $\tau_{\text {Ross }}=\tau_{0} \mathrm{e}^{-r / R_{0}+1}$, where $\tau_{0}$ is the opacity at a some radial point $R_{0}$. The horizontal dashed lines marks $\tau_{\text {Ross }}=1$. Points below this line are usually associated with too shallow initial structures. which is proportional to the kinetic-energy injection, and compare with the wind properties. We find that, in general,

$\log (\dot{M}) \propto \log (q)$

while the wind velocity $u_{\text {out }}$ shows a more complicated dependence on $q$ (see Figs. 2 and 3). The latter is caused by the levitation of the outer atmospheric layers and the increase in density, which affects the dust-formation efficiency.

In Figs. 2 and 3, we note that the B-models with metalpoor composition, show typically faster winds than solar metallicity B-models, and that metal-poor A-models show mass-loss rates higher than the corresponding models with solar composition (see Fig. 2). We note also that the mass-loss rates and mean degrees of dust condensation ( $f_{\mathrm{c}}$-values), obtained for the A-models with metal-poor composition, in principle, show no trends with $q$; in these cases, this is because the region of wind formation is saturated with dust, i.e., $f_{\mathrm{c}}$ reaches its upper limit (typically $\sim 0.4-0.5$ ), and the wind is maximally dust-driven. Furthermore, the metal-poor A-models with $\Delta u_{\mathrm{p}} \lesssim 2 \mathrm{~km} \mathrm{~s}^{-1}$ cannot sustain a wind due to inefficient dust formation. The same is true for B-models with $\Delta u_{\mathrm{p}} \lesssim 3 \mathrm{~km} \mathrm{~s}^{-1}$. This is because the temperature gradient is slightly flatter in the AGS05 models, which affects the conditions for dust formation negatively and therefore requires a higher kinetic-energy input to trigger dust formation.

\subsection{The $\dot{M}-q$ connection}

From the above, it is obvious that there is a connection between the average mass-loss rate and the $q$-parameter. In principle we find three different cases: $\dot{M} \propto q^{1 / 2}, \dot{M} \propto q$, and $\dot{M}=$ const., which can be derived from the equation of motion for the atmosphere and wind; a detailed discussion is beyond the scope of this letter and will be published in a forthcoming paper. Before we discuss the consequences (see Sect. 3.3), it is of interest to discuss how these dependences on $q$ arise from a physical point of view.

The first two cases reflect the effects of the energy-input by pulsations, which affects the levitation of the outer atmospheric layers and mean degree of dust condensation. Hence, the massloss rate increases as more kinetic energy is pumped into the atmosphere. The third trend, or the absence of a trend, is due to saturation of the dust-formation mechanism, i.e., there is an upper limit for the dust/gas-ratio. When this limit is reached, the dust opacity does not change when more kinetic energy is pumped into the atmosphere and the mass-loss rate remains constant. The transitions between these cases depend on the period/luminosity of the model stars, and their atmospheric structures.

\subsection{Stronger winds at lower metallicity?}

As is evident in Figs. 2 and 3, a larger $q$-value corresponds, in general, to a larger mass loss and the models with a metal-poor composition have typically faster winds and higher mass-loss rates for given stellar parameters. They also require larger $q$ values to sustain a wind. In the case of the A-models, the trends (mass-loss rates as a function of $q$ ) appear to be different for the two metallicities considered, although the number of models is too small to establish the existence of two different trends statistically. However, in all cases, our modelling results suggest that the winds may become stronger at sub-solar metallicities, or at least not weaker than at solar metallicity, provided that the abundance of free carbon is the same even if the over all metallicity is different. This result may explain observations of mass-loss rates of C-stars in the LMC, and other local dwarf galaxies (van Loon et al. 2000, 2003; Matsuura et al. 2007), in which the mass-loss rates appear to be as high, or even higher, than for C-stars in the solar neighbourhood. Similar results were obtained by Jackson et al. $(2007 \mathrm{a}, \mathrm{b})$ for $\mathrm{C}$-stars in other dwarf galaxies.

\section{Summary and conclusions}

The results presented above may be summarised as follows:

The mass loss of C-rich AGB-stars, for a given set of stellar parameters and abundance of free carbon, is a function of the energy-injection by pulsations as long as the dust formation has not reached the saturation limit. When this limit is reached, the mass-loss rate no longer increases with the energy-injection.

The range of realistic, or even possible, pulsation energies is limited, and connected to the stellar parameters. The treatment of the inner boundary and pulsations in the case of a non-saturated wind is crucial. Hence, the importance of constraints on the energy injection by pulsations cannot be emphasised enough. A quantitatively-correct theory, or reliable observational constraints, of long-period stellar pulsation are required to obtain a complete quantitative understanding of mass loss on the AGB. Since the results here are based on only a rather small grid of models, further study of boundary-condition effects is needed and will be presented in a forthcoming paper. 

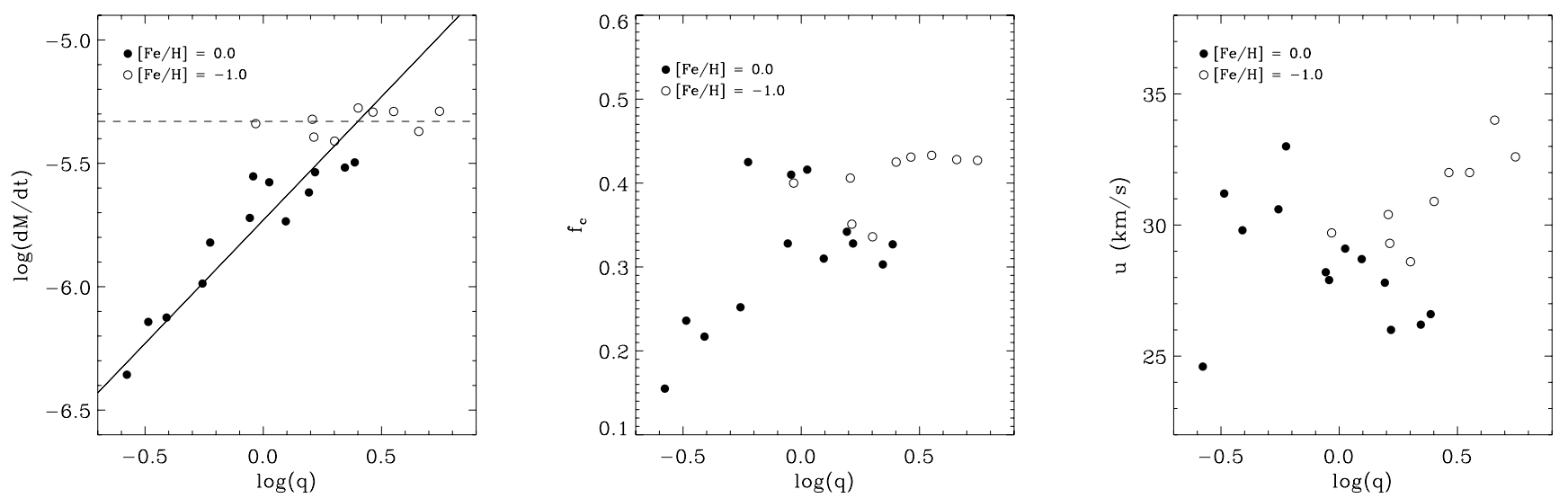

Fig. 2. Comparison between mass-loss rate, mean degree of dust condensation $\left(f_{\mathrm{c}}\right)$, and wind velocity for A-models, where $\log (\tilde{\varepsilon})=8.8($ see also Table 2), as functions of the energy-injection parameter $q$ for solar (Asplund et al. 2005) and a typical metal-poor composition $([\mathrm{Fe} / \mathrm{H}]=-1.0)$. The solid and dashed lines in the left panel indicate $\langle\dot{M}\rangle \propto q$ and $\langle\dot{M}\rangle=$ const., respectively.
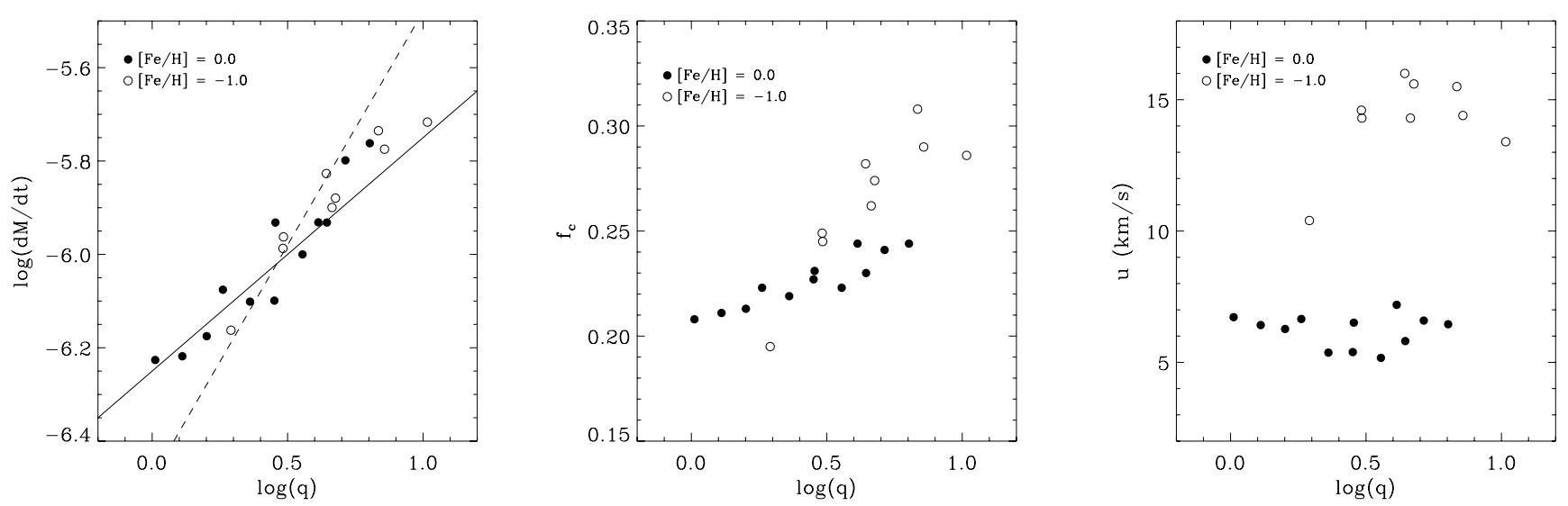

Fig. 3. Comparison between mass-loss rate, mean degree of dust condensation $\left(f_{\mathrm{c}}\right)$, and wind velocity for B-models, where $\log (\tilde{\varepsilon})=8.5$ (see also Table 2), as functions of the energy-injection parameter $q$ for solar (Asplund et al. 2005) and a typical metal-poor composition $([\mathrm{Fe} / \mathrm{H}]=-1.0)$. The solid and dashed lines in the left panel indicate $\langle\dot{M}\rangle \propto q^{1 / 2}$ and $\langle\dot{M}\rangle \propto q$, respectively.

However, one firm conclusion can be drawn from present results: low metallicity should not necessarily be associated with a lower mass-loss rate. More precisely, the development of a strong super-wind phase is expected for C-stars at low (subsolar) metallicity. The duration of the AGB-phase may, in particular, be affected and the effects on nucleosynthesis results (stellar yields) may be profound.

Acknowledgements. The authors wish to thank the referee, J.Th. van Loon, for his careful reading of the manuscript and constructive criticism that helped to improve the clarity of this paper. B. Gustafsson is thanked for his critical reading and valuable comments on the manuscript. A. Wachter is thanked for making unpublished results on mass loss at low metallicity available to us.

\section{References}

Andersen, A. C., Höfner, S., \& Gautschy-Loidl, R. 2003, A\&A, 400, 981 Asplund, M., Grevesse, N., Sauval, A. J. 2005, ASPC, 336, 25A

Bowen, G. H., \& Willson, L.-A. 1991, ApJ, 375, 53

Feast, M. W., Glass, I. S., Whitelock, P. A., \& Catchpole, R. M. 1989, MNRAS, 241,375
Gautschy-Loidl, R., Höfner, S., Jorgensen, U. G., \& Hron, J. 2004, A\&A, 422, 289

Groenewegen, M., Wood, P. R., Sloan, G. C., et al. 2007, MNRAS, 376, 313

Helling, C., Winters, J. M., \& Sedlmayer, E. 2000, A\&A, 358, 651

Herwig, F. 2005. ARA\&A, 43, 435

Höfner, S., Gautschy-Loidl, R., Aringer, B., \& Jørgensen, U. G. 2003, A\&A, 399,589

Jackson, D. C., Skillman, E. D., Gerhrz, R. D., et al. 2007a, ApJ, 656, 818

Jackson, D. C., Skillman, E. D., Gerhrz, R. D., et al. 2007b, ApJ, 667, 891

Lagadec, E., Zijlstra, A. A., Sloan, G. C., et al. 2007, MNRAS, 376, 1270 van Loon, J. Th. 2000, A\&A, 354, 125

van Loon, J. Th., Marshall, J. R., Matsuura, M., \& Zijlstra, A. A. 2003, MNRAS, 341,1205

van Loon J.Th., 2008, [arXiv: 0712 . 2754]

Matsuura, M., et al. 2007, MNRAS, 382, 1889

Olivier, E. A., \& Wood, P. R. 2005, 362, 1396

Wachter, A., Schröder, K.-P., Winters, J. M., et al. 2002, A\&A, 384, 452

Wachter, A., Winters, J. M., Schröder, K.-P., \& Sedlmayr, E. 2008, A\&A, submitted

Wahlin, R., Eriksson, K., Gustafsson, B., et al. 2006, Mem.S.A.It., 77, 955

Westerlund, B. E., Azzopardi, M., Breysacher, J., \& Rebeirot, E. 1991, A\&AS, 91,425

Winters, J. M., Le Bertre, T., Jeong, K. S., Helling, C. \& Sedlmayr, E. 2000, A\&A, 361, 641

Wood, P. 1979, ApJ, 227, 220 